Pacific Journal of Mathematics

GENERALIZED ORDERED SPACES WITH CAPACITIES

BenNett and David John Lutzer 


\title{
GENERALIZED ORDERED SPACES WITH CAPACITIES
}

\author{
H. R. BENNETT AND D. J. LUTZER
}

\begin{abstract}
We show that any $G O$-space having a capacity in the sense of Ščepin has a $G_{\delta}$-diagonal and is perfect. In addition, such a space has a $\sigma$-discrete dense subset and a dense metrizable subspace, and any $G O$ space having a capacity and a point-countable base (or having a $\sigma$-discrete dense subset and a point-countable base) is metrizable.
\end{abstract}

1. Introduction. In [14] Ščepin defined a capacity for a space $X$ to be a family of functions $\left\{\varepsilon_{x} \mid x \in X\right\}$ such that, for each closed $F \subset X$,

$\left(C_{1}\right) \varepsilon_{x}(F)$ is a non-negative real number with $\varepsilon_{x}(F)>0$ iff $x \in$ $\operatorname{Int}(F)$,

$\left(\mathrm{C}_{2}\right)$ if $F_{1} \subset F_{2}$ are closed then $\varepsilon_{x}\left(F_{1}\right) \leq \varepsilon_{x}\left(F_{2}\right)$,

$\left(\mathrm{C}_{3}\right)$ for a fixed closed $F$, the function $x \rightarrow \varepsilon_{x}(F)$ is continuous,

$\left(\mathrm{C}_{4}\right)$ for a fixed $x$, if $\left\{F_{\alpha} \mid \alpha<\kappa\right\}$ is a family of closed sets satisfying $F_{\alpha} \supset F_{\beta}$ whenever $\alpha<\beta<\kappa$, then $\varepsilon_{x}\left(\cap_{\alpha} F_{\alpha}\right)=\inf _{\alpha} \varepsilon_{x}\left(F_{\alpha}\right)$.

In that same paper Ščepin announced without proof that a linearly ordered topological space (LOTS) having a capacity is metrizable. The purpose of this note is to prove a more general result from which Ščepin's result follows immediately, namely, that any $G O$-space (= suborderable space) with a capacity has a $G_{\delta}$-diagonal. (Recall that the class of $G O$-spaces is precisely the class of subspaces of LOTS.) Along the way to that result, we show that any $G O$-space with a capacity is perfect (i.e., closed sets are $\left.G_{\delta}\right)$. In $\S 4$ we will discuss two old questions about perfect $G O$-spaces in the context of $G O$-spaces having a capacity, proving that a $G O$-space with a capacity has a $\sigma$-discrete dense subset and a $G O$-space with a capacity and a point-countable base must be metrizable. Finally, examples in $\S 5$ show that our results are sharp.

Terminology and notation not defined in this paper will follow $[8, \mathbf{1 1}$, 12].

2. Preliminary results and perfect normality. We proceed via a sequence of lemmas.

2.1. Lemma. Any GO-space having a capacity is a first-countable space. 
Proof. Fix a non-isolated point $p$ of $X$. If $[p, \rightarrow)$ is not open then $\varepsilon_{p}[p, \rightarrow)=0$ and there is a well-ordered, strictly increasing net $\left\{x_{\alpha} \mid \alpha<\kappa\right\}$ whose supremum is $p$. Let $F_{\alpha}=\left[x_{\alpha}, \rightarrow\right)$. According to $\left(\mathrm{C}_{4}\right), 0=$ $\varepsilon_{p}([p, \rightarrow))=\inf \left\{\varepsilon_{p}\left(F_{\alpha}\right) \mid \alpha<\kappa\right\}$. For each $n$, choose $\alpha_{n}<\kappa$ such that $\alpha_{n-1}<\alpha_{n}$ and $\varepsilon_{p}\left(F_{\alpha_{n}}\right)<1 / n$. If some point $y$ of $X$ has $x_{\alpha_{n}} \leq y<p$ for each $n$, then for each $n$ we have $0<\varepsilon_{p}([y, \rightarrow))<\varepsilon_{p}\left(\left[x_{\alpha_{n}}, \rightarrow\right)\right)<1 / n$, which is impossible. Hence $p$ is the limit of a sequence $z_{n}=x_{\alpha_{n}}$ from $(\leftarrow, p)$. If $(\leftarrow, p]$ is open, then $\left\{\left(z_{n}, p\right] \mid n \geq 1\right\}$ is a neighborhood base at $p$. If $(\leftarrow, p]$ is not open, we can obtain a sequence $w_{1}>w_{2}>\cdots$ having $p$ as its limit, and then $\left\{\left(z_{n}, w_{n}\right) \mid n \geq 1\right\}$ is a local base at $p$. Other cases are handled analogously.

\subsection{Proposition. Any GO-space with a capacity is perfect.}

Proof. Let $U$ be any open set and let $\mathcal{V}=\left\{V_{\alpha} \mid \alpha \in A\right\}$ be the family of all convex components of $U$. For each $\alpha \in A$ choose $p_{\alpha} \in V_{\alpha}$. Then $\varepsilon_{p_{\alpha}}\left(\bar{V}_{\alpha}\right)>0$. Let $P_{n}=\left\{p_{\alpha} \mid \varepsilon_{p_{\alpha}}\left(\bar{V}_{\alpha}\right) \geq 1 / n\right\}$. We claim that $P_{n}$ is a closed, discrete subspace of $X$. Obviously $P_{n}$ is discrete-in-itself. We show $P_{n}$ is closed. Let $q$ be a limit point of $P_{n}$. Since $X$ is first-countable, there is a strictly monotonic sequence $\left\langle q_{k}\right\rangle$ from $P_{n}$ whose limit is $q$, say $q_{k}=p_{\alpha_{k}}$. Let $F=\{q\} \cup\left(\cup\left\{\bar{V}_{\alpha_{2 k}} \mid k \geq 1\right\}\right)$. Then $F$ is a closed set and, by $\left(\mathrm{C}_{3}\right)$, $\varepsilon_{q}(F)=\lim _{k \rightarrow \infty} \varepsilon_{q_{2 k}}(F) \geq 1 / n$ because $\varepsilon_{q_{2 k}}(F) \geq \varepsilon_{q_{2 k}}\left(\bar{V}_{\alpha_{2 k}}\right) \geq 1 / n$. Hence $q \in \operatorname{Int}(F)$. But the sequence $\left\{q_{2 k+1} \mid k \geq 1\right\}$ also converges to $q$ and no term of that sequence lies in $F$, contradicting $q \in \operatorname{Int}(F)$. Hence $P_{n}$ is closed and discrete.

Since $X$ is first countable, each set $V_{\alpha} \in \mathcal{T}$ is an $F_{\sigma}$-set so we may find closed convex sets $D(\alpha, k)$ having $p_{\alpha} \in D(\alpha, 1) \subset D(\alpha, 2) \subset \cdots$ and $\cup\{D(\alpha, k) \mid k \geq 1\}=V_{\alpha}$. Let $E(n, k)=\bigcup\left\{D(\alpha, k) \mid p_{\alpha} \in P_{n}\right\}$. Since $P_{n}$ is closed and discrete, each $E(n, k)$ is closed, and $U=\cup \mathcal{V}=$ $\cup\{E(n, k) \mid n \geq 1, k \geq 1\}$.

REMARK. Corollary 4.3 below provides an even stronger conclusion than does Proposition 2.2.

2.3. Lemma. Suppose $(\leftarrow, p]$ is not open. Let $\delta>0$. Then there is a point $q>p$ such that for each $t \in[p, q], \varepsilon_{t}([p, q])<\delta$.

Proof. Since $p$ is a limit point of $(p, \rightarrow)$ there is a sequence $b_{1}>b_{2}>$ $\cdots$ whose limit is $p$. Then $0=\varepsilon_{p}((\leftarrow, p])=\inf \left\{\varepsilon_{p}\left(\left(\leftarrow, b_{n}\right]\right) \mid n \geq 1\right\}$ so that for some $n_{0}, \varepsilon_{p}\left(\left(\leftarrow, b_{n_{0}}\right]\right)<\delta$. Then $\varepsilon_{p}\left(\left[p, b_{n_{0}}\right]\right)<\delta$. Now assume no point $q$, as described in the Lemma, exists. Let $c_{0}=b_{n_{0}}$. Then there is a 
point $t_{0} \in\left[p, c_{0}\right]$ with $\varepsilon_{t_{0}}\left(\left[p, c_{0}\right]\right) \geq \delta$. Necessarily, $p<t_{0}$. Let $c_{1}=$ $\min \left\{b_{n_{0}+1}, t_{0}\right\}$ and find $t_{1} \in\left[p, c_{1}\right]$ with $\varepsilon_{t_{1}}\left(\left[p, c_{1}\right]\right) \geq \delta$. In general, find a point $t_{k+1} \in\left[p, c_{k+1}\right]$ with $\varepsilon_{t_{k+1}}\left(\left[p, c_{k+1}\right]\right) \geq \delta$, where $c_{k+1}=$ $\min \left\{b_{n_{0}+k+1}, t_{k}\right\}$. If $m$ is fixed and $k>m, p<c_{k}<c_{m}$ and so $\varepsilon_{t_{k}}\left(\left[p, c_{m}\right]\right)$ $\geq \varepsilon_{t_{k}}\left(\left[p, c_{k}\right]\right) \geq \delta$. Letting $k \rightarrow \infty$, we obtain $\varepsilon_{p}\left(\left[p, c_{m}\right]\right)=\lim _{k} \varepsilon_{t_{k}}\left(\left[p, c_{m}\right]\right)$ $\geq \delta$. But $c_{m} \leq b_{n_{0}+m}<b_{n_{0}}$ so we obtain $\delta \leq \varepsilon_{p}\left(\left[p, c_{m}\right]\right) \leq \varepsilon_{p}\left(\left[p, b_{n_{0}}\right]\right)<\delta$, a contradiction.

REMARK. There is an obvious analogue of $(2.3)$ in case $[p, \rightarrow)$ is not open.

2.4. LemMA. Suppose neither $(\leftarrow, p]$ nor $[p, \rightarrow)$ is open (i.e., $p$ is a two-sided limit point of $X)$. Let $\delta>0$. Then there are points $q$ and $r$ with $q<p<r$ having the property that for every $t \in[q, r], \varepsilon_{t}([q, r])<\delta$.

Proof. The proof is analogous to the proof of (2.3).

2.5. Notation. Let $(X, \mathcal{T},<)$ be a $G O$-space. Let

$R=\{x \in X \mid[x, \rightarrow)$ is open $\}$,

$L=\{x \in X \mid(\leftarrow, x]$ is open $\}$,

$I=\{x \in X \mid\{x\}$ is open $\}$,

$R^{*}=R-I$, and

$L^{*}=L-I$.

2.6. LemMA. Assume $X$ is a GO-space having a capacity. Each of the sets defined in (2.5) is an $F_{\sigma}$-set.

Proof. In the light of (2.2), $I$ is an $F_{\sigma}$-set since $I$ is open. If we can show that $R$ is an $F_{\sigma}$-set, then so is $R^{*}$ because $R^{*}=R-I$.

To show that $R$ is an $F_{\sigma}$-set, observe that for each $x \in R, \varepsilon_{x}([x, \rightarrow))$ $>0$. Let $R_{n}=\left\{x \in R \mid \varepsilon_{x}([x, \rightarrow)) \geq 1 / n\right\}$. Suppose $p$ is a limit point of $R_{n}$. Choose a strictly monotonic sequence $\left\langle x_{k}\right\rangle$ from $R_{n}$ whose limit is $p$. There are two cases.

Case 1. Suppose $x_{1}<x_{2}<\cdots$. Then $[p, \rightarrow)=\bigcap\left\{\left[x_{k}, \rightarrow\right) \mid k \geq 1\right\}$ so that $\varepsilon_{p}([p, \rightarrow))=\inf \left\{\varepsilon_{p}\left(\left[x_{k}, \rightarrow\right)\right) \mid k \geq 1\right\}$. If $k$ is fixed and $m>k$ then $x_{k}<x_{m}$ so that $\varepsilon_{x_{m}}\left(\left[x_{k}, \rightarrow\right)\right) \geq \varepsilon_{x_{m}}\left(\left[x_{m}, \rightarrow\right)\right) \geq 1 / n$. Letting $m \rightarrow \infty$, we obtain $\varepsilon_{p}\left(\left[x_{k}, \rightarrow\right)\right)=\lim \varepsilon_{x_{m}}\left(\left[x_{k}, \rightarrow\right)\right) \geq 1 / n$. Hence $\varepsilon_{p}([p, \rightarrow)) \geq 1 / n$. But then $p$ must be an interior point of $[p, \rightarrow)$ so that the increasing sequence $\left\langle x_{k}\right\rangle$ could not have converged to $p$. 
Case 2. Suppose $x_{1}>x_{2}>\cdots$. According to $\left(\mathrm{C}_{3}\right), \varepsilon_{p}([p, \rightarrow))=$ $\lim _{k} \varepsilon_{x_{k}}([p, \rightarrow))$. Since $p<x_{k}, \varepsilon_{x_{k}}([p, \rightarrow)) \geq \varepsilon_{x_{k}}\left(\left[x_{k}, \rightarrow\right)\right) \geq 1 / n$. Hence $\varepsilon_{p}([p, \rightarrow)) \geq 1 / n$. But then $p$ must be an interior point of $[p, \rightarrow)$ so that $p \in R$. Hence $p \in R_{n}$ as required.

Analogously, $L$ and $L^{*}$ are $F_{\sigma}$ sets.

3. $G_{\delta}$-diagonals. Ceder [6] observed that the diagonal of space $X$ is a $G_{\delta}$-subset of $X \times X$ if there are open coverings $\mathcal{G}(n)$ of $X$ (for $n \geq 1$ ) such that given $x \neq y$ in $X, \operatorname{St}(x, \mathcal{G}(n)) \subset X-\{y\}$ for some $n$. In perfect spaces, a weaker condition suffices. The proof of the next lemma is easy.

3.1. Lemma. Suppose $X$ is perfect. Then $X$ has a $G_{\delta}$-diagonal if there is a countable family $\Psi$ such that

(a) each $\mathcal{G} \in \Psi$ is a collection of open subsets of $X$, and,

(b) given $x \neq y$ in $X$, some $\mathcal{G} \in \Psi$ has $x \in \operatorname{St}(x, \mathcal{G}) \subset X-\{y\}$.

3.2. Lemma. Suppose $X$ is a GO-space with a capacity. Then there is a countable family $\Psi_{R}$ such that

(a) each $\mathcal{G} \in \Psi_{R}$ is a collection of open subsets of $X$, and,

(b) given $x \in R$ and $y \neq x$, some $\mathcal{G} \in \Psi_{R}$ has $x \in \operatorname{St}(x, \mathcal{G}) \subset X-\{y\}$.

Proof. Let $\mathcal{G}_{0}=\{\{x\} \mid x \in I\}$. For $n \geq 1$ and for $p \in R^{*}$, use Lemma (2.3) to find a point $q(p, n)>p$ such that for every $t \in[p, q(p, n)]$, $\varepsilon_{t}([p, q(p, n)])<1 / n$. Let $\mathcal{G}(n)=\left\{[p, q(p, n)) \mid p \in R^{*}\right\}$. Next, use Lemma (2.6) to write $L=\bigcup\left\{L_{k} \mid k \geq 1\right\}$ where each $L_{k}$ is closed in $X$, and notice that $R^{*} \cap L=\varnothing$. Now define, for $n \geq 1, \mathcal{G}(-n)=\left\{X-L_{n}\right\}$. We let $\Psi_{R}=\{\mathcal{G}(n) \mid n$ is any integer $\}$.

Fix $x \in R$ and $y \neq x$. If $x \in I$, then $\operatorname{St}(x, \mathcal{G}(0))=\{x\} \subset X-\{y\}$ as required, so assume $x \in R-I=R^{*}$. Let $J$ be the convex hull of the two-point set $\{x, y\}$. There are two cases.

Case 1. If there is some point $t$ having $\varepsilon_{t}(J)>0$, find a positive integer $n$ having $\varepsilon_{t}(J)>1 / n$. Since $x \in R^{*},[x, q(x, n)) \in \mathcal{G}(n)$ so that $x \in \operatorname{St}(x, \mathcal{G}(n))$. Suppose some member $[p, q(p, n))$ of $\mathcal{G}(n)$ contains both $x$ and $y$. By convexity, $J \subset[p, q(p, n)]$ so we have $\varepsilon_{t}(J) \leq$ $\varepsilon_{t}([p, q(p, n)])$. But $t \in[p, q(p, n)]$ so that $1 / n>\varepsilon_{t}([p, q(p, n)]) \geq$ $\varepsilon_{t}(J)>1 / n$, which is impossible. Hence $y \notin \operatorname{St}(x, \mathcal{G}(n))$.

Case 2. If there is no point $t$ in $X$ such that $\varepsilon_{t}(J)>0$, then $y<x$, because if $x<y$ we would have $[x, y)=[x, \rightarrow) \cap(\leftarrow, y)$, so $x$ would be an interior point of $J$, whence $\varepsilon_{x}(J)>0$. Since $y<x$ and since no point $t$ 
of $X$ lies strictly between $x$ and $y$, we conclude that $(\leftarrow, y]=(\leftarrow, x)$ is open. Thus $y \in L$. Choose $n$ so that $y \in L_{n}$. Because $R^{*} \cap L_{n}=\varnothing$, $x \in \operatorname{St}(x, \mathcal{G}(-n))=X-L_{n} \subset X-\{y\}$, as required.

3.3. Remark. Suppose $X$ is a $G O$-space with a capacity. There is an analogue of (3.2) which constructs a countable family $\Psi_{L}$ of open collections such that if $x \in L$ and $y \in X-\{x\}$, then some $\mathcal{G} \in \Psi_{L}$ has $x \in$ $\operatorname{St}(x, \mathcal{G}) \subset X-\{y\}$.

3.4. Lemma. Suppose $X$ is a GO-space with a capacity. Let $E=X-$ $(R \cup L \cup I)$. Then there is a countable family $\Psi_{E}$ such that

(a) each $\mathcal{G} \in \Psi_{E}$ is a collection of open subsets of $X$, and,

(b) if $x \in E$ and if $y \in X-\{x\}$, then for some $\mathcal{G} \in \Psi_{E}, x \in \operatorname{St}(x, \mathcal{G})$ $\subset X-\{y\}$.

Proof. For each $p \in E$, use Lemma (2.4) to select points $a(p, n)<p$ $<b(p, n)$ such that for each $t \in[a(p, n), b(p, n)], \varepsilon_{t}([a(p, n), b(p, n)])$ $<1 / n$. For $n \geq 1$, let $\mathcal{G}(n)=\{(a(p, n), b(p, n)) \mid p \in E\}$, and let $\Psi_{E}=$ $\{\mathcal{G}(n) \mid n \geq 1\}$. The proof that $\Psi_{E}$ satisfies (b) above is similar to, but even easier than, the proof that $\Psi_{R}$ satisfies (b) of (3.2).

3.5. THEOREM. Any GO-space with a capacity has a $G_{\delta}$-diagonal.

Proof. Using the collections found in (3.2)-(3.4) let $\Psi=\Psi_{R} \cup \Psi_{L} \cup$ $\Psi_{E}$. Then $\Psi$ satisfies the hypotheses of (3.1) so that, since $X$ is perfect in the light of (2.2), $X$ has a $G_{\delta}$-diagonal.

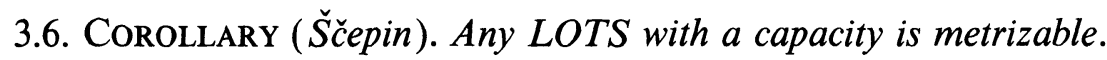

Proof. Any LOTS with a $G_{\delta}$-diagonal is metrizable [10].

4. Some results on perfect spaces. There are two old questions which concern perfect $G O$-spaces. The first is due to R. W. Heath, and the second was posed by M. Maurice and J. van Wouwe.

(H) Find a real example of a perfect $G O$-space which has a pointcountable base and yet is not metrizable.

$(\mathrm{MvW})$ Find a real example of a perfect $G O$-space which does not have a $\sigma$-discrete dense subset.

(These questions ask for "real examples", i.e., examples in ZFC, since if there is a Souslin line, then there is a counterexample to each [2], [13], [15].) 
In this section we show that no counterexample to $(\mathrm{H})$ or to $(\mathrm{MvW})$ can have a capacity.

It is known that any $G O$-space having a $\sigma$-discrete dense subset is perfect [15]. We begin this section by proving the converse for $G O$-spaces having a capacity, thereby strengthening (2.2). We need the following result, due to Przymusiński [1].

4.1. Proposition. Let $(X, \mathcal{T},<)$ be a GO-space having a $G_{\delta}$-diagonal. Then there is a topology \% on $X$ such that:

(a) ( $X$, গ ) is metrizable;

(b) $\Re \subset \mathcal{T}$;

(c) $(X, \Re,<)$ is a GO-space.

4.2. Theorem. Suppose $X$ is a perfect $G O$-space having a $G_{\delta}$-diagonal. Then $X$ has a $\sigma$-discrete dense subset.

Proof. Let $\mathcal{T}$ and $<$ be, respectively, the topology and ordering of $X$. Use (4.1) to find a metrizable GO-topology $\mathfrak{T} \subset \mathcal{T}$. Let $D$ be a $\sigma$-discrete dense subset of the metric space $(X, \Re)$ and let $I=\{x \mid\{x\} \in \mathscr{T}-\Re\}$. Then $D$ is also $\sigma$-discrete in $(X, \mathcal{T})$ and $I$ is an $F_{\sigma}$ in $(X, \mathcal{T})$, whence $I$ is also $\sigma$-discrete in $(X, \mathcal{T})$. Let $E=D \cup I$.

Now let $W$ be any nonvoid open set. If $W \cap I \neq \varnothing$ then $W \cap E \neq \varnothing$, so assume $W$ contains no isolated points. Then there are points $a<b$ in $W$ such that $\varnothing \neq(a, b) \subset W$. But then $(a, b) \in \Re$ so $(a, b) \cap D \neq \varnothing$. Hence $W \cap E \neq \varnothing$, as required.

4.3. Corollary. Any GO-space with a capacity has a $\sigma$-discrete dense set.

Proof. Combine (2.2), (3.5) and (4.2).

4.4. Corollary. Any GO-space with a capacity has a dense metrizable subspace.

Proof. The $\sigma$-discrete dense set $D$ found in (4.3) is, in its relative topology, semistratifiable in the sense of Creede [7] and any semistratifiable $G O$-space is metrizable [11]. 
To show that no counterexample to $(\mathrm{MvW})$ can have a capacity we prove a bit more, namely:

4.5. THEOREM. Let $X$ be a GO-space having a $\sigma$-discrete dense set and $a$ point-countable base. Then $X$ is metrizable.

Proof. Since any $G O$-space having a $\sigma$-discrete dense set is perfect and paracompact [15], it will be enough to show that a space $X$ which satisfies the hypotheses of (4.5) has a $\sigma$-disjoint base. Then $X$ is quasi-developable [3] and perfect, so $X$ is developable [3]. But a developable paracompact space is metrizable.

Let $D=\bigcup\{D(n) \mid n \geq 1\}$ be a $\sigma$-discrete dense subset of $X$. A standard argument [Prop. 3.4, 5] provides a $\sigma$-disjoint base for points of $D$. Let $I$ be the set of isolated points of $X$ (so $I \subset D$ ). Let $R^{*}$ and $L^{*}$ be as in (2.5) and let $E=X-\left(R^{*} \cup L^{*} \cup I\right)$. A standard argument shows that the collection $\mathscr{V}=\cup\left\{\mathcal{V}_{n} \mid n \geq 1\right\}$, where $\mathcal{V}_{n}$ is the collection of convex components of $X-D(n)$, contains a $\sigma$-disjoint base for all points of $E$. Therefore it suffices to find $\sigma$-disjoint collections $\mathcal{C}$ and $\mathcal{C}^{\prime}$ which contain neighborhood bases for all points of $R^{*}-D$ and $L^{*}-D$, respectively. We show how to find $\mathrm{C}$.

Let $\Re$ be a point-countable base for $X$, and let $\mathscr{V}=\cup\left\{\mathscr{V}_{n} \mid n \geq 1\right\}$ be as above. For $n \geq 1$ and $V \in \mathcal{V}_{n}$, let $\mathscr{P}_{n}(V)=\{B \cap V \mid B \in \mathscr{B}$ and for some $\left.p \in R^{*} \cap V,([p, \rightarrow) \cap V) \subset B \subset[p, \rightarrow)\right\}$. Let $\mathscr{P}_{n}=\cup\left\{\mathscr{P}_{n}(V)\right\}$ $\left.V \in \mathscr{V}_{n}\right\}$ and $\mathscr{P}=\cup\left\{\mathscr{P}_{n} \mid n \geq 1\right\}$. Then we have

1. $\mathscr{P}$ is point-countable, and

2. $P$ contains a neighborhood base at each point of $R^{*}-D$.

Fix $n$ and $V \in \mathscr{V}_{n}$. For each $P \in \mathscr{P}_{n}(V)$ there is a unique $y_{P} \in P \cap V$ having $P=\left[y_{P}, \rightarrow\right) \cap V$. Let $C(n, V)=\left\{y_{P} \mid P \in \mathscr{P}_{n}(V)\right\}$ and choose $S(n, V)=\{x(V, \alpha) \mid \alpha<\kappa(V)\}$, a cofinal strictly increasing subset of $C(n, V)$. Because $\mathscr{P}_{n}(V)$ is point-countable, we have

3. If $\alpha<\kappa(V)$ then $|C(n, V) \cap(\leftarrow, x(V, \alpha))| \leq \omega_{0}$. For each $y \in C(n, V)$, let $\alpha(n, V, y)$ be the first index $\beta<\kappa(V)$ such that $y<x(V, \beta)$ and define

$$
\mathcal{C}(n, V, \alpha)=\{[y, x(V, \alpha)) \mid y \in C(n, V) \text { and } \alpha(n, V, y)=\alpha\} \text {. }
$$

If $V \neq W$ belong to $\mathscr{V}(n)$ or if $V=W$ and $\alpha \neq \beta$, then $\mathcal{C}(n, V, \alpha) \cap$ $\mathcal{C}(n, W, \beta)=\varnothing$. Furthermore,

4. each $\mathcal{C}(n, V, \alpha)$ is countable. 
Index $e(n, V, \alpha)$ as $\{C(n, V, \alpha, k) \mid k \geq 1\}$ and let $e^{\prime}(n, k)=$ $\left\{C(n, V, \alpha, k) \mid V \in \mathcal{V}_{n}, \alpha<\kappa(V)\right\}$. Then we have

5. the family $\mathcal{C}=\cup\left\{\mathcal{C}(n, V, \alpha) \mid n \geq 1, V \in \mathscr{V}_{n}\right.$, and $\left.\alpha<\kappa(V)\right\}$ has $\mathcal{C}=\cup\left\{\mathcal{C}^{\prime}(n, k) \mid n \geq 1, k \geq 1\right\}$, so that $\mathrm{C}_{\text {is } \sigma \text {-disjoint. }}$

It remains only to show that $C$ contains a neighborhood base at each point of $R^{*}-D$. Fix $p \in R^{*}-D$ and $r>p$. Find $B \in \Re$ with $p \in B \subset$ $[p, r[$. Because $p \notin I$ we may find $q>p$ with $[p, q) \subset B \subset[p, r)$ and $(p, q) \neq \varnothing$. Choose $n$ so that $(p, q) \cap D(n) \neq \varnothing$ and choose $d \in(p, q)$ $\cap D(n)$. Because $p \in R-D$, some convex component $V \in \mathcal{T}_{n}$ contains $p$. Then $V \subset(\leftarrow, d)$ and so

$$
p \in[p, \rightarrow) \cap V \subset[p, \rightarrow) \cap(\leftarrow, d) \subset[p, d) \subset[p, q) \subset B \subset[p, \rightarrow),
$$

i.e., the set $Q=B \cap V$ belongs to $\mathscr{P}_{n}(V)$. The unique point $y_{Q}$ with $Q=\left[y_{Q}, \rightarrow\right) \cap V$ is $y_{Q}=p$, so $p \in C(n, V)$. Compute $\alpha=\alpha(n, V, p)$. Then $[p, x(V, \alpha)) \in \mathcal{C}(n, V, \alpha) \in \mathcal{C}$ and $[p, x(V, \alpha)) \subset Q \subset B \subset[p, r)$. Hence $C$ contains a neighborhood base at each point of $R^{*}-D$, as required.

4.6. Corollary. Any GO-space having a capacity and a point-countable base is metrizable.

Theorem 2.1 of [4] shows that a perfect $G O$-space with a $\delta \theta$-base has a point-countable base. Hence we have:

4.7. Corollary. Any GO-space having a capacity and a $\delta \theta$-base is metrizable.

We conclude this section by pointing out that, in the light of (4.5), any counterexample for $(\mathrm{H})$ is also a counterexample of the type required in $(\mathrm{MvW})$.

\section{Examples.}

5.1 It is easy to see that the Sorgenfrey line [3] has a capacity. Thus, Theorem (3.5) cannot be strengthened to assert that a $G O$-space with a capacity is metrizable.

5.2 No uncountable subspace of the Michael line $[3,11]$ can have a capacity unless it is metrizable. For if $X$ is an uncountable subspace of the Michael line, then $X$ is quasi-developable since it has a $\sigma$-disjoint base [11]. If $X$ had a capacity then $X$ would be perfect (2.2) and perfect quasi-developable space is developable [3]. But a developable $G O$-space is metrizable. (We remark that, under $(\mathrm{MA}+\neg \mathrm{CH})$, there are uncountable 
subsets of the Michael line $M$ which are metrizable; indeed Theorem (4.1) of [9] shows that any subspace $X$ of $M$ with $|X|<c$ is metrizable.)

5.3 It is not true that a perfect $G O$-space with a $G_{\delta}$-diagonal and a $\sigma$-discrete dense set must have a capacity. Let $X$ be the $G O$-space obtained from the usual real line $\mathbf{R}$ by making the half-line $[x, \rightarrow$ ) open whenever $x$ is irrational and using the usual open interval neighborhoods for rational numbers. Then $X$ is separable and has a $G_{\delta}$-diagonal. However the set $R=\{x \in X \mid[x, \rightarrow)$ is open $\}$ is not an $F_{\sigma}$-subset of $X$, so $X$ does not have a capacity.

\section{REFERENCES}

[1] K. Alster, Subparacompactness in Cartesian products of generalized ordered topological spaces, Fund. Math., 87 (1975), 7-28.

[2] H. Bennett, On quasi-developable spaces, Ph.D. Thesis Arizona State University, 1968.

[3] _ On quasi-developable spaces, Gen. Top. Appl., 1 (1971), 253-262.

[4] , GO-spaces with $\delta \theta$-bases, Topology and Order Structures, I, M.C. Tract 142, Mathematical Center, Amsterdam, 1981.

[5] H. Bennett and D. Lutzer, Ordered spaces with $\sigma$-minimal bases, Topology Proc., 2 (1977), 371-382.

[6] J. Ceder, Some generalizations of metric spaces, Pacific J. Math., 11 (1961), 105-125.

[7] G. Creede, Concerning semistratifiable spaces, Pacific J. Math., 32 (1970), 47-54.

[8] R. Engelking, General Topology, Polish Scientific Publishers, 1977.

[9] W. Fleissner and G. M. Reed, Paralindelöf spaces and spaces with a $\sigma$-locally countable base, Topology Proc., 2 (1977), 89-110.

[10] D. Lutzer, A metrization theorem for linearly orderable spaces, Proc. Amer. Math. Soc., 22 (1969), 557-558.

[11] __ On generalized ordered spaces, Dissertations Math., 89, 1971.

[12] __ Ordered Topological Spaces, Surveys in General Topology, ed. by G. M. Reed, Academic Press, New York, 1980.

[13] V. Ponomarev, Metrizability of a finally compact p-space with a point-countable base, Sov. Math. Dokl., 8 (1967), 765-768.

[14] E. Ščepin, On topological products, groups, and a new class of spaces more general than metric spaces, Soviet Math. Dokl., 17 (1976), 152-155.

[15] J. van Wouwe, GO-spaces and generalizations of metrizability, M.C. Tract 104, Mathematical Center, Amsterdam, 1979.

Received May 14, 1982. The second author was partially supported by NSF Grant MCS 80-01617.

TEXAS TECH UNIVERSITY

LUBBOCK, TX 79409

AND

MiAMI UNIVERSITY

OXFORD, OH 45056 



\section{PACIFIC JOURNAL OF MATHEMATICS \\ EDITORS}

DONALD BABBITT (Managing Editor)

University of California.

Los Angeles, CA 90024

Hugo Rossi

University of Utah

Salt Lake City, UT 84112

C. C. Moore and Arthur Ogus

University of California

Berkeley, CA 94720
J. DugundjI

Department of Mathematics

University of Southern California

Los Angeles, CA 90089-1113

R. FinN and H. Samelson

Stanford University

Stanford, CA 94305

\section{ASSOCIATE EDITORS}
R. ARENS
E. F. BECKENBACH
B. H. NeumanN
F. WOLF
K. YoshidA (1906-1982)

\section{SUPPORTING INSTITUTIONS}

UNIVERSITY OF ARIZONA

UNIVERSITY OF BRITISH COLUMBIA

CALIFORNIA INSTITUTE OF TECHNOLOGY

UNIVERSITY OF CALIFORNIA

MONTANA STATE UNIVERSITY

UNIVERSITY OF NEVADA. RENO

NEW MEXICO STATE UNIVERSITY

OREGON STATE UNIVERSITY
UNIVERSITY OF OREGON

UNIVERSITY OF SOUTHERN CALIFORNIA

STANFORD UNIVERSITY

UNIVERSITY OF HAWAII

UNIVERSITY OF TOKYO

UNIVERSITY OF UTAH

WASHINGTON STATE UNIVERSITY

UNIVERSITY OF WASHINGTON 


\section{Pacific Journal of Mathematics}

Vol. 112, No. $1 \quad$ January, 1984

Richard Blaine Barrar and Henry Loeb, Characterizing the divided difference weights for extended complete Tchebycheff systems $\ldots \ldots \ldots \ldots 1$

Harold Bennett and David John Lutzer, Generalized ordered spaces with

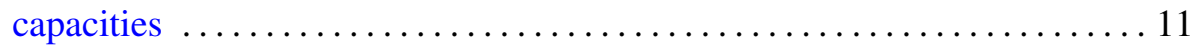

Geoffrey J. Butler and Lynn Harry Erbe, Comparison theorems for second-order operator-valued linear differential equations

Bohumil Cenkl and Richard D. Porter, de Rham theorem with cubical

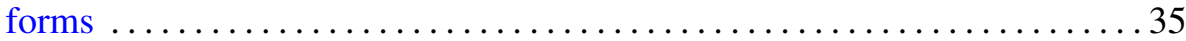

Zvonko Cerin, Characterizing global properties in inverse limits . ....... 49

Thomas Eugene Duchamp and Morris Kalka, Holomorphic foliations and deformations of the Hopf foliation .........................69 69

John Paul Hempel, Homology of coverings $\ldots \ldots \ldots \ldots \ldots \ldots \ldots \ldots \ldots$

Gerald Norman Hile and R. Z. Yeh, Inequalities for eigenvalues of the

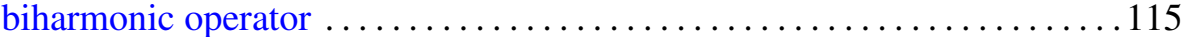

Kenneth Irwin Joy, A description of the topology on the dual space of a

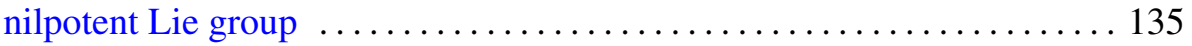

Alex Kumjian, On localizations and simple $C^{*}$-algebras $\ldots \ldots \ldots \ldots \ldots 141$

Bernardus de Pagter, The space of extended orthomorphisms in a Riesz

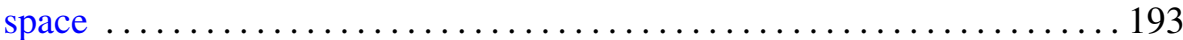

Stephen C. Persek, Iterated averaging for periodic systems with hidden

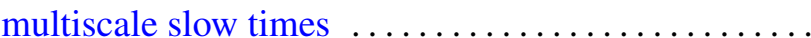

David Francis Rearick, Divisibility of arithmetic functions . . . . . . . . . 237

Masaaki Suzuki, The intrinsic metrics on the circular domains in $\mathbf{C}^{n}$ 